\title{
Some recent discoveries in roAp stars
}

\author{
D.W. Kurtz ${ }^{1}$, V.G. Elkin ${ }^{1}$, G. Mathys ${ }^{2}$, J. Riley ${ }^{1}$, M.S. Cunha ${ }^{3}$, \\ H. Shibahashi ${ }^{4}$ and E. Kambe ${ }^{5}$ \\ ${ }^{1}$ Centre for Astrophysics, University of Central Lancashire, Preston PR1 2HE, UK \\ e-mail: dwkurtz@uclan.ac.uk \\ ${ }^{2}$ European Southern Observatory, Casilla 19001, Santiago 19, Chile \\ ${ }^{3}$ Centro de Astrofsica da Universidade do Porto, Rua das estrelas, 4150 Porto, Portugal \\ ${ }^{4}$ Department of Astronomy, University of Tokyo, Tokyo 113-0033, Japan \\ ${ }^{5}$ Department of Earth and Ocean Sciences, National Defence Academy, Yokosuka, Kanagawa \\ 239-8686, Japan
}

\begin{abstract}
Research in roAp stars is being vigorously pursued, both theoretically and observationally by many groups. We report the discovery of a 21 -min period, luminous roAp star, HD 116114. Longer periods for more luminous stars have been predicted theoretically and this is the first discovery of such a star. We discuss a model for the blue-to-red line profile variability observed in some roAp stars involving a shock wave high in the atmosphere of roAp stars, yet show that the $\mathrm{H} \alpha$ line in $33 \mathrm{Lib}$ has the blue-to-red-to-blue line profile variability expected for subsonic dipolar pulsation concentrated towards the pulsation pole. Further we report for $33 \mathrm{Lib}$ unprecedented observations of the amplitudes and phases of its principal mode at $2.015 \mathrm{mHz}$ and its first harmonic of that at $4.030 \mathrm{mHz}$.
\end{abstract}

Keywords. Stars: chemically peculiar, stars: oscillations, stars: variables: other, stars: individual: (HD 116114, HD 137949)

\section{Introduction}

The roAp stars have been observed photometrically since their discovery by Kurtz (1982) over 20 years ago. Frequency analyses of their light curves have yielded rich asteroseismic information on the degrees of the pulsation modes, distortion of the modes from normal modes, magnetic geometries, and luminosities. The latter, in particular, are derived asteroseismically and agree well with Hipparcos luminosities (Matthews et al. 1999).

Theoretical work on the interaction of pulsation with both rotation and the magnetic field by Bigot \& Dziembowski (2002) has presented a new look at the oblique pulsator model of these stars. They find that the pulsation axis is inclined to both the magnetic and the rotation axes, and the pulsation modes are complex combinations of spherical harmonics that result in modes that, in many cases, can be travelling waves looking similar to (but are not exactly) sectoral m-modes. Bigot \& Kurtz (2004) have shown that the improved oblique pulsator model of Bigot \& Dziembowski yields the rotational inclination and the magnetic obliquity for the roAp star HR 3831 that are in excellent agreement with those found from magnetic studies, whereas the old oblique pulsator model is in probable disagreement with the magnetic studies. Kochukhov (2005), however, finds very different rotational and magnetic inclinations for HR 3831 from the Doppler mapping of the pulsation mode. He suggests that the magnetic geometry needs to be reconsidered in this light, and his geometry is consistent with the original oblique pulsator model and the new model of Saio \& Gautschy (2004), who find modes that are aligned 
with the magnetic axis and are distorted by the magnetic field so that they cannot be described by single spherical harmonics. They note that the horizontal motion can be comparable to the vertical motion for these modes. It is the unique geometry of the pulsation modes in roAp stars that allows us to examine their non-radial pulsation modes from varying aspects as can be done with no other type of star. At present there is a great deal of research, both observational and theoretical, on these stars, with some exciting conflicts that will be resolved in time.

The spectra of many roAp stars show a strong core-wing anomaly in the Hydrogen lines, particularly the $\mathrm{H} \alpha$ line (Cowley et al. 2001, Kochukhov et al. 2002). This indicates abnormal atmospheric structure, as does that consistent abundances for the second and third ionisation states of Rare Earth elements, particularly Nd and Pr, have not yet been found for these stars (Ryabchikova et al. 2002). Until atmospheric models can be found that solve these problems, caution is called for, but new high-resolution spectroscopic results for the roAp stars suggest vertical stratification of some ions, particularly the Rare Earths, and show the short vertical wavelengths of the pulsation modes. It must also be cautioned that there are known horizontal abundance variations with the concentration of the Rare Earth elements towards the magnetic poles. See, for example, studies of $\gamma$ Equ (HD 201601) (Kochukhov \& Ryabchikova 2001a), HD 166473 (Kurtz et al. 2003), $\alpha$ Cir (Kochukhov \& Ryabchikova 2001b, Balona \& Laney 2003, Baldry et al. 1999), HR 3831 (Kochukhov \& Ryabchikova 2001b, Baldry \& Bedding 2000), HR 1217 (Balona \& Zima 2002, Sachkov et al. 2004) and 33 Lib (HD 137949) (Mkrtichian et al. 2003, Kurtz et al. 2004). The results of these studies are plausibly interpreted in terms of the chemical stratification of the elements in the atmospheres of these stars. In general, Fe lines originate near a radial node around optical depth $\tau=1$ with little, or no radial velocity variations seen. The core of $\mathrm{H} \alpha$ forms higher in the atmosphere and shows radial velocity variations with amplitudes of hundreds of $\mathrm{ms}^{-1}$. Lines from the first and second ionisation states of the Rare Earths Pr and Nd arise from a thin layer around optical depth $\tau=10^{-3}$ and can show amplitudes of $\mathrm{km} \mathrm{s}^{-1}$.

The spectroscopic studies have begun a three-dimensional resolution of the pulsation modes, with the vertical stratification giving depth information, the rotation of the oblique mode providing information on the surface geometry of the modes, and, in the case of HR 3831, Doppler imaging of the pulsation mode giving two-dimensional geometrical information (Kochukhov 2005). While these spectroscopic tools are very powerful, they demand high spectroscopic resolution, high time resolution and high signal-to-noise $(S / N)$, requirements that can only be met with large telescopes. It is thus not possible at present to study in detail the frequencies in roAp stars spectroscopically because of the need for high duty cycle data sets over extended periods of time. Photometric studies are still required to obtain asteroseismic frequency spectra.

\section{2. roAp versus noAp stars: HD 116114}

Hubrig et al. (2000) compared the positions of the roAp stars and noAp (nonoscillating Ap) stars in the HR diagram and concluded that the roAp stars are, on average, closer to the Main Sequence than the noAp stars. This has important implications for the stilluncertain driving mechanism for the roAp stars. There has been a question, however, about whether a selection effect could have caused this apparent separation of the roAp and noAp stars. Most of the 33 known roAp stars were discovered by Martinez and Kurtz photometrically in various studies. They preferentially searched for stars with strong Strömgren $\delta m_{1}$ indices and $\delta c_{1}$ indices that are negative, indicating strong line blanketing. For the coolest Ap stars a negative $\delta c_{1}$ index is often an indicator of an 
extremely peculiar star, and such stars are often found to be roAp stars. However, $\delta c_{1}$ increases with luminosity so that an extremely peculiar evolved cool Ap star may show a normal $\delta c_{1}$. The surveys of Martinez and Kurtz would have been much less likely to find roAp stars among such stars, so there is a selection effect in their discovery.

Cunha (2002) predicted an instability strip for roAp stars and found that the more evolved stars should pulsate with longer periods, in some cases in the range of $20-25 \mathrm{~min}$. Again, the high-speed photometric searches of Martinez and Kurtz could easily miss variability on this timescale because of confusion with sky transparency variations, since they usually searched for only $1-2 \mathrm{hr}$ per star when surveying for new roAp stars. To find longer periods of the order predicted by Cunha photometric searches should be for $3-4 \mathrm{hr}$ to try to separate the pulsation signal from low frequency sky transparency noise. This demands the most stable photometric conditions.

As it turns out, the discovery of a longer-period roAp star has come from a spectroscopic radial velocity study. Elkin et al. (2004) have found the cool Ap star HD 116114 to be pulsating with a period near $21 \mathrm{~min}$, a value close to that predicted by Cunha (2002) for a star of the luminosity and temperature of HD 116114. This discovery found radial velocity variations with amplitudes of only tens of $\mathrm{ms}^{-1}$ in certain selected lines in the spectrum of HD 116114. Such precision is possible with the high resolution VLT UVES data obtained for this star. Fig. 1 shows an amplitude spectrum for 11 spectral lines with the pulsation signal clearly visible at $\nu=0.79 \mathrm{mHz}(P=21 \mathrm{~min})$.

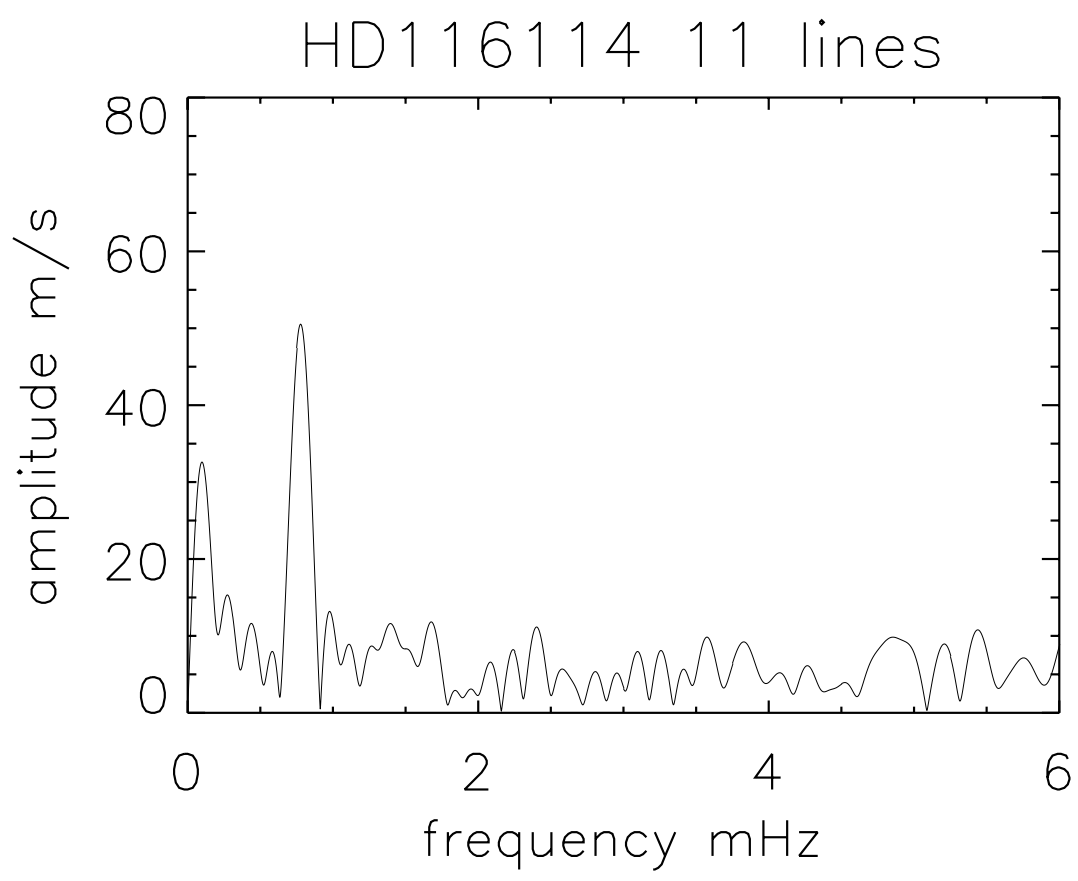

Figure 1. Amplitude spectrum of the radial velocity variations for 11 spectral lines in the Ap star HD 116114. The $50 \mathrm{~m} \mathrm{~s}^{-1}$ signal at $0.79 \mathrm{mHz}(P=21 \mathrm{~min})$ is clear.

One implication of this discovery is that there may be more luminous roAp stars with longer periods as predicted by Cunha (2002). A search is now being undertaken to discover more of these stars and study them in some detail. If they are found in some numbers, then the luminosity difference between the roAp and noAp stars may disappear, 
leaving an understanding of why some Ap stars pulsate and some do not more mysterious.

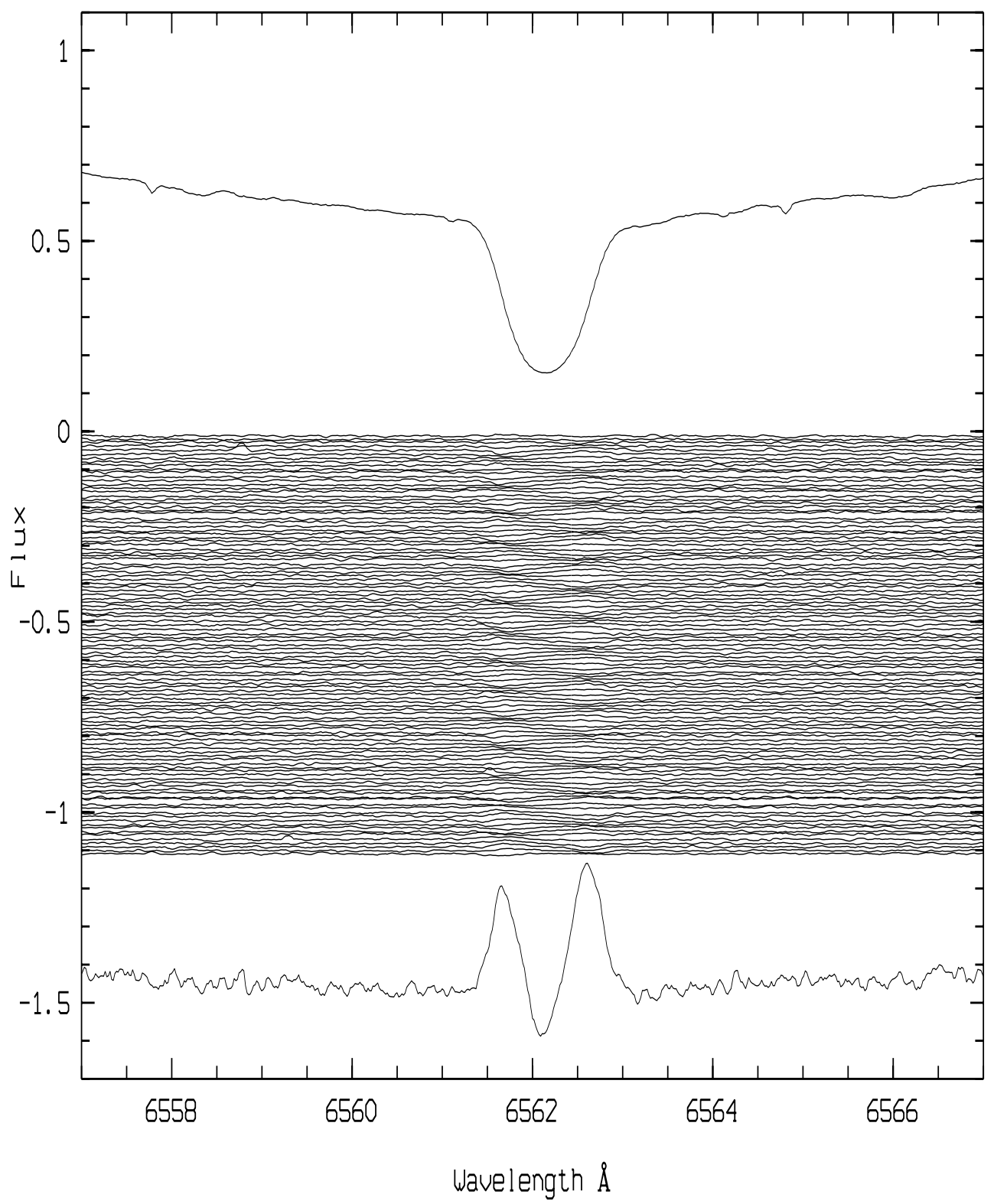

Figure 2. The H $\alpha$ LPV in $33 \mathrm{Lib}$. The blue-to-red-to-blue pattern is as expected for subsonic pulsation for a dipole mode concentrated towards the pulsation pole, as is seen in model LPV in Fig. 3.

\section{Line profile variability in roAp stars}

Line profile variations (LPV) in $\gamma$ Equ showing apparent blue-to-red travelling bumps were discovered by Kochukhov \& Ryabchikova (2001a). Shibahashi et al. (2004) proposed 
that these line profile variations are a manifestation of a shock wave in the high atmosphere near the magnetic polar regions. This idea was vigorously discussed at IAUS224. Other stars with similar profile variations have now been discovered by several groups working on these stars.

An example is $33 \mathrm{Lib}$ (see, e.g., Kurtz et al. 2004). For this star the LPV for Nd III $\lambda 6145$, which forms high in the atmosphere of the star, has the same apparent blue-to-red bumps seen in $\gamma$ Equ. The $\mathrm{H} \alpha$ line, however, shows a blue-to-red-to-blue, back-and-forth LPV as can be seen in Fig. 2. This is as expected for radial mode pulsation, or for dipole pulsation with the variation concentrated to the pulsation pole, where the velocities are subsonic. We show this in model LPV in Fig. 3 for a dipole concentrated towards the pulsation pole.

The important point is that the maximum speed must be faster than the atmospheric sound speed for the lines that show only blue-to-red LPV. The pulsation amplitude in velocity is expected to increase with the decrease of the density with height. So the amplitude in velocity naturally exceeds the sound speed at a certain level, and a shock wave is generated. When this shock wave propagates through the layer under consideration, the layer is suddenly kicked upward by the shock wave and then falls down after reaching the peak height, at which the speed of the layer is zero. This process repeats and the resultant variation in the Doppler shift is qualitatively in agreement with the observed one in Nd III lines of $\gamma$ Equ and 33 Lib. The lack of variation in the standard deviation in the middle of each cycle occurs as the layer comes to a stop, then falls back.

This picture may also be consistent with the mode identifications from the photometric observations, which imply axisymmetric dipole modes whose symmetry axis coincides with the magnetic axis, both being inclined to the rotation axis of the star. If it is correct, then the following are expected: 1) The LPV should be sinusoidal for the spectral lines formed in layers where the wave motion is subsonic as seen for $\mathrm{H} \alpha$ in $33 \mathrm{Lib}$ in Fig. 2, 2) The LPV should be sinusoidal for chemical elements that are not concentrated in the magnetic polar regions, so their pulsation velocities remain subsonic, and 3) The LPV should show monotonic blue-to-red motion only for the lines formed in the high atmosphere near the magnetic polar regions.

\section{Atmospheric depth dependence of pulsation in $33 \mathrm{Lib}$}

For the roAp stars that have been observed at high spectral resolution few generalities of the radial velocity behaviour, beyond those given above, can yet be made. Kurtz, Mathys and Elkin have now obtained high time resolution $(\leqslant 1 \mathrm{~min})$, high spectral resolution $(\mathrm{R}=105000)$ and high signal-to-noise ratio (50-600) spectra for two hours each for 15 known roAp stars (including HD 116114 which was discovered with this data set) and 6 other roAp candidates and sharp-lined A stars using the UVES spectrograph on UT2 (Kueyen) of the VLT on Paranal, Chile.

One of our targets, $33 \mathrm{Lib}$ (HD 137949), is a bright $(V=6.68)$ roAp that is known photometrically to have a principal pulsation frequency of $2.015 \mathrm{mHz}(P=8.27 \mathrm{~min})$ with a first harmonic frequency at $4.030 \mathrm{mHz}$ (Kurtz 1991). We have analysed a set of 111 echelle spectra of 33 Lib obtained with the UVES spectrograph on UT2 (Kueyen) of the VLT. Each spectrum had an integration time of $40 \mathrm{~s}$ with the readout and overhead time between spectra of $25 \mathrm{~s}$, giving a time resolution of $65 \mathrm{~s}$. The typical signal-to-noise $(S / N)$ ratio is about 200 and the time span of the observations is $2 \mathrm{hr}$.

33 Lib has three pulsation modes that can be seen in our 2-hour duration data set, the known 2.015-mHz mode and harmonic at $4.030 \mathrm{mHz}$, and a new, low-amplitude frequency at $1.769 \mathrm{mHz}$ that we will not discuss here. The largest amplitudes found for the principal 
$\mathrm{SPH} L=1 \mathrm{M}=0 \mathrm{AMP}=0.4 \mathrm{~km} / \mathrm{s} \quad \mathrm{C}=4 \mathrm{~km} / \mathrm{s} \quad \mathrm{ncl}=0$

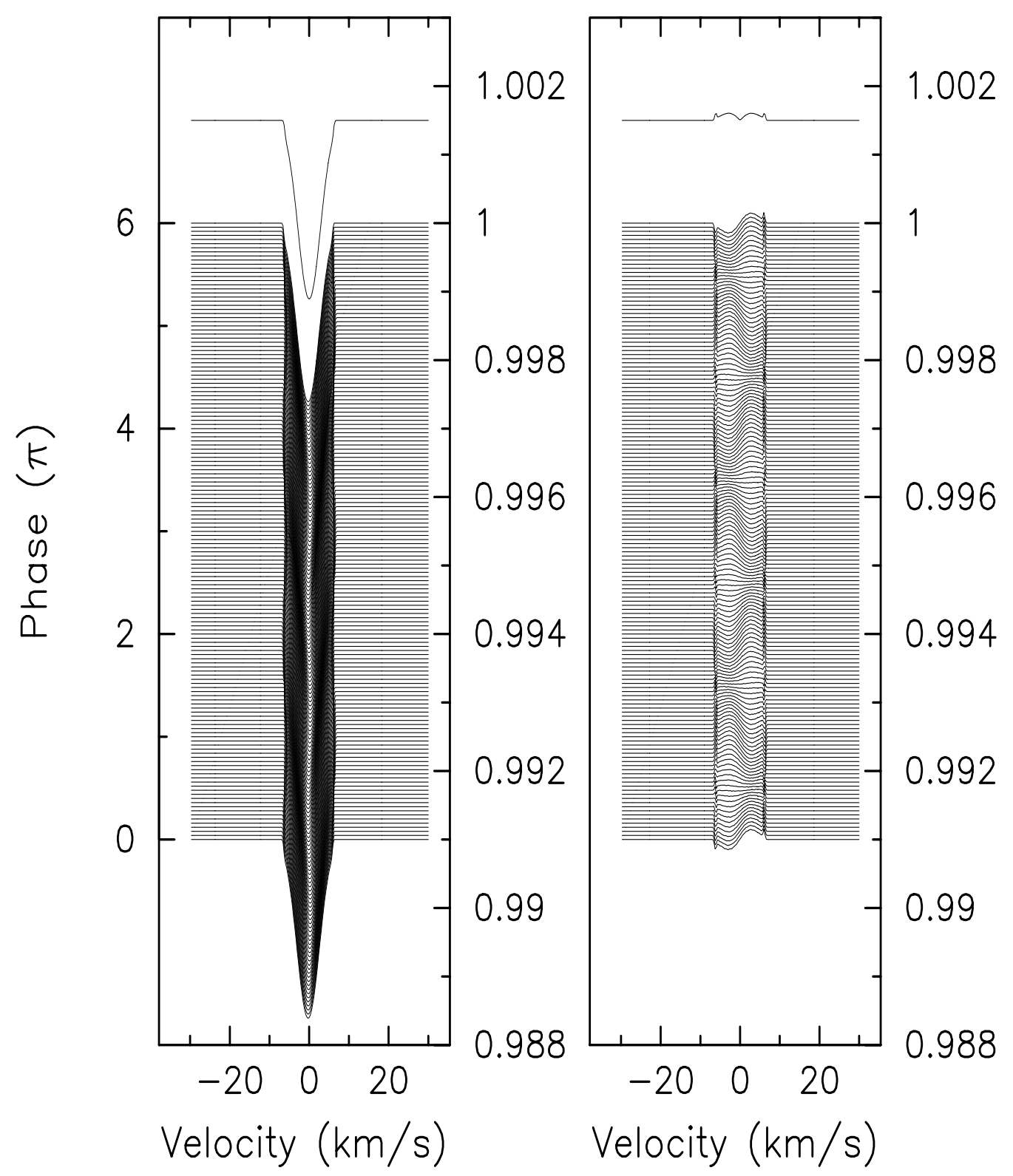

Figure 3. Theoretically expected LPV of an $\ell=1, m=0$ mode for a non-rotating star. The pulsation amplitude is assumed to be $0.4 \mathrm{~km} \mathrm{~s}^{-1}$ and the line profile is assumed to have an intrinsic line width corresponding to a velocity of $4 \mathrm{~km} \mathrm{~s}^{-1}$. The aspect angle between the pulsation axis and the line-of-sight is assumed to be $0^{\circ}$. For each mode the LPV for one pulsation cycle are shown on the left panel, and the difference between the LPV and the average profile (shown top left) are displayed in the right panel. The LPV move from blue to red and back again, as is observed for the $\mathrm{H} \alpha$ line in $33 \mathrm{Lib}$ as shown in Fig. 2. 
pulsation frequency are for $\mathrm{H} \alpha$ and some lines of singly-ionised Rare Earth elements, Nd II, Ce II and Sm II with semi-amplitudes of $200-300 \mathrm{~m} \mathrm{~s}^{-1}$. Lines of Nd III, Pr III and Eu II typically have amplitudes in the $120-200 \mathrm{~m} \mathrm{~s}^{-1}$ range, while lines of $\mathrm{Fe}, \mathrm{Cr}$ and Ca usually have amplitudes less than $40 \mathrm{~m} \mathrm{~s}^{-1}$. Fig. 4 shows the amplitude spectra for Nd II $\lambda 5319.815$ and Nd III $\lambda 6145.070$ showing all three of these frequencies. The apparent independent behaviour of the $4.030-\mathrm{mHz}$ peak led Kurtz et al. (2004) to suggest that it is an independent mode, rather than an harmonic of the $2.015-\mathrm{mHz}$ peak. We are working on an interpretation of it as an harmonic, even so, this harmonic behaves in many ways like an independent mode.
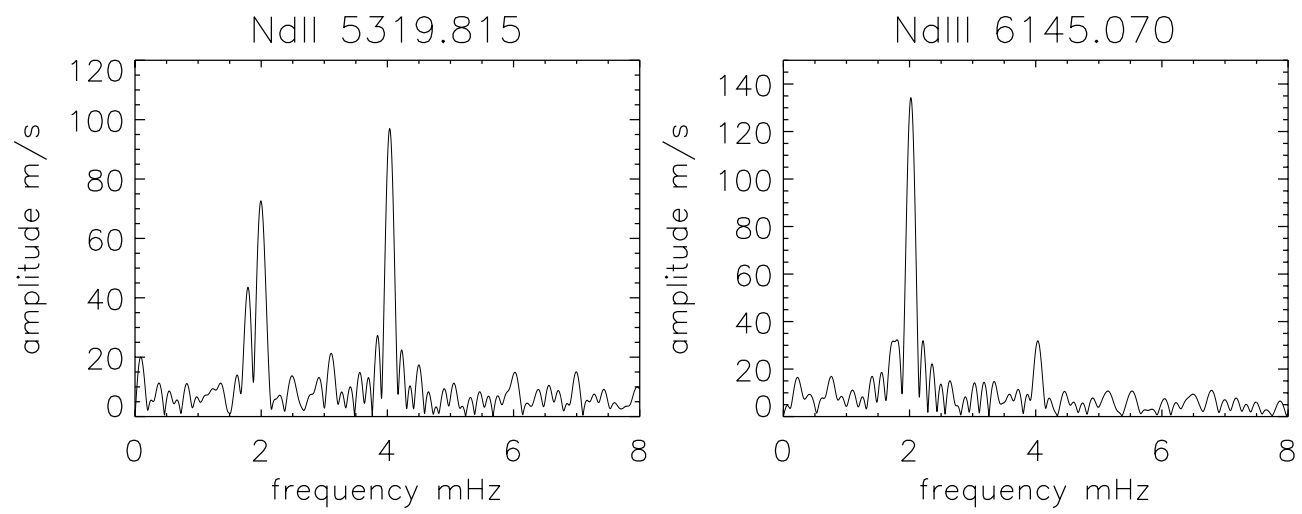

Figure 4. Left panel: The amplitude spectrum of the radial velocity variations for Nd II $\lambda 5319.815$. The known principal frequency at $2.015 \mathrm{mHz}$ and the new frequency at $1.769 \mathrm{mHz}$ are resolved at a separation of $246 \mu \mathrm{Hz}$. The highest amplitude mode is at the harmonic frequency $4.030 \mathrm{mHz}$. Note the absence of any second harmonic. Compare this with Nd III $\lambda 6145.070$ in the right panel, showing the independence of the amplitudes of the $2.015-\mathrm{mHz}$ and $4.030-\mathrm{mHz}$ frequencies.
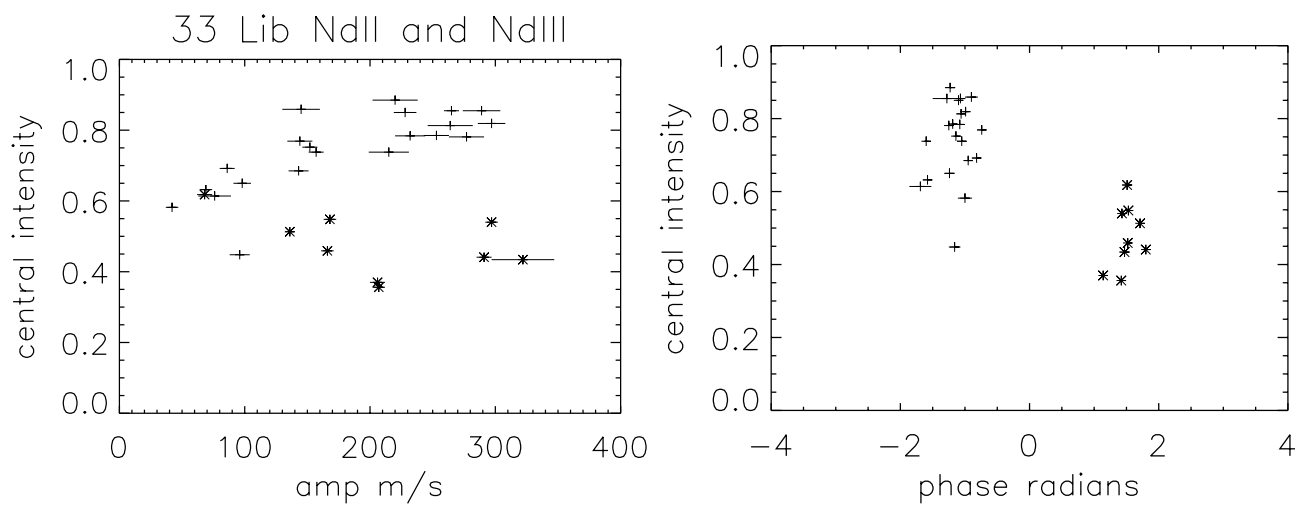

Figure 5. The amplitudes and phases of the Nd II and Nd III lines as a function of central line intensity normalised to 1 at the continuum. The smaller the value of the central intensity, the higher in the atmospheric layer for that ion the line forms. The left panel shows the amplitudes for the Nd II lines (plus signs) and Nd III lines (asterisks). Because of decreasing density and ionisation equilibrium, the Nd III lines form higher in the atmosphere than the Nd II lines. The two layers lie on opposite sides of a radial pulsation node with the Nd III amplitudes increasing outwards away from the node, and the Nd II amplitudes increasing inwards away from the node. The phases suggest standing waves which differ by nearly $\pi$ radians.

Fig. 5 shows the amplitudes and phases determined by least squares for the 2.015$\mathrm{mHz}$ mode for $\mathrm{Nd}$ II and NdIII. For each of these ions the phases do not appear to 
vary as a function of atmospheric depth. Those phases differ between the ions by $2.65 \pm$ 0.31 radians, nearly in antiphase ( $\pi$ radians) as found by Mkrtichian et al. (2003). The new discovery is the clear increase in amplitude with atmospheric height for Nd III and the increase in amplitude with atmospheric depth for Nd II. This is completely consistent with Mkrtichian et al.'s suggestion that the lines of these two ions form on opposite sides of a pulsation node with Nd III above, and Nd II below the node.
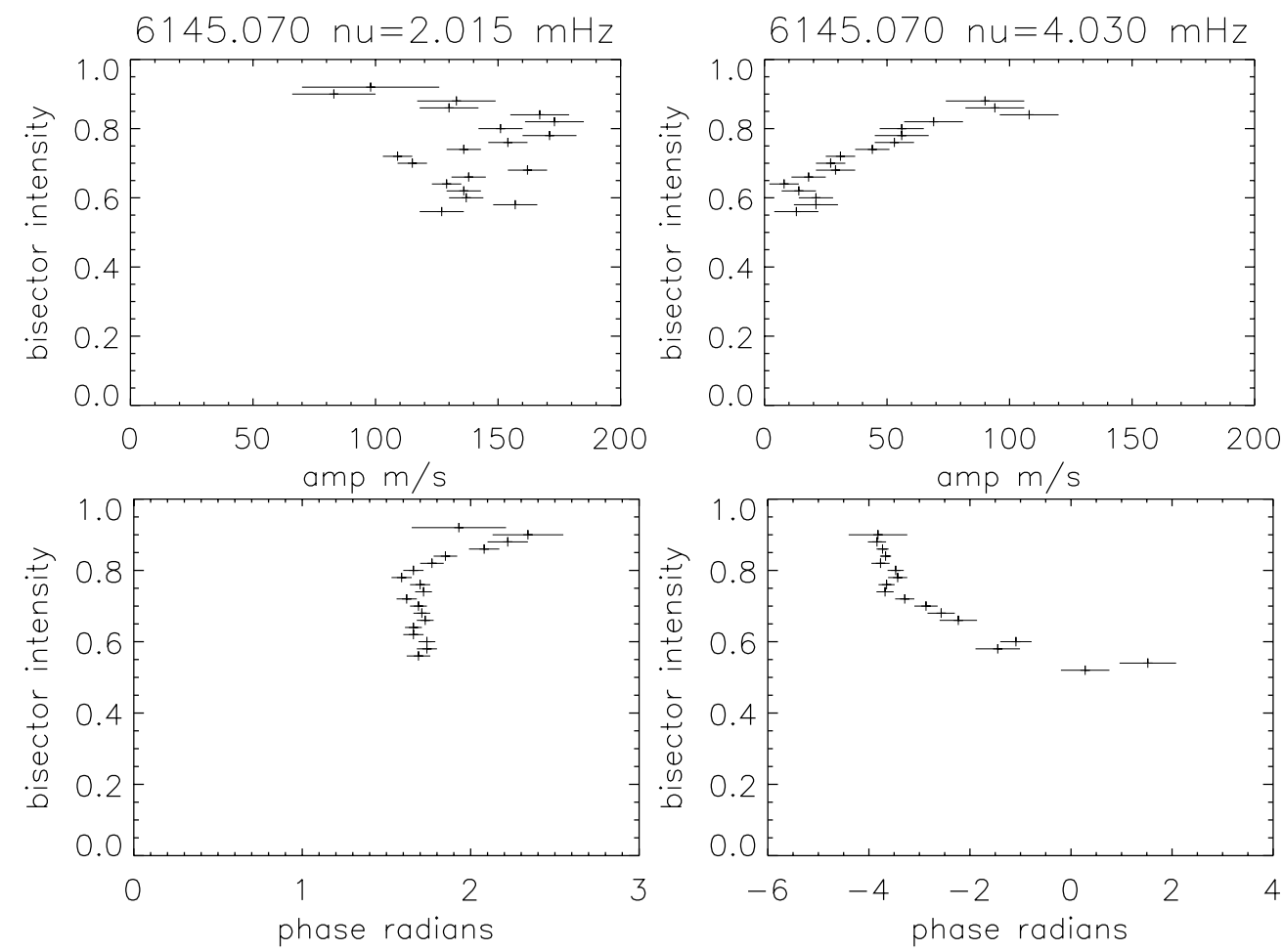

Figure 6. The amplitudes and phases for Nd III $\lambda 6145.070$ at central intensity 0.56 to 0.92 in steps of 0.02 (where 1 is the continuum) for the $2.015-\mathrm{mHz}$ mode on the left and for the $4.030-\mathrm{mHz}$ harmonic on the right. The deeper the line depth (weaker the central intensity), the higher in the atmosphere the cut through the line is formed. The phases have been calculated using the function $\cos (2 \pi f t+\phi)$, so the times of maxima occur earlier for larger phases.

The constancy of the phases for this mode suggests standing waves on both sides of the node, but there is an unprecedented new way (for any star other than the Sun) to examine the pulsation amplitudes and phases as a function of atmospheric height. We have done that by making cuts through the strong line $\mathrm{Nd}$ III $\lambda 6145.070$ at central intensity 0.56 to 0.92 in steps of 0.02 (continuum $=1$ ). We have measured the radial velocities of the line bisector at each of those depths, and then fitted by linear least squares the $2.015-\mathrm{mHz}$ and $4.030-\mathrm{mHz}$ frequencies to extract amplitudes, phases and their associated errors. The results are consistent with a constant radial velocity amplitude in the Nd III layer for the $2.015-\mathrm{mHz}$ mode, although an increase with height is possible. There also seems to be a standing wave higher in the layer with a running wave travelling outwards from below. This is not what is expected of a purely acoustic mode. For that we expect a standing wave with an outwardly running wave above it where energy is being dissipated in the boundary layer. We do not know, this is the first observation of such behaviour, and 
theoretical studies (Saio \& Gautschy 2004, Cunha \& Gough 2000, Bigot et al. 2000) do not yet address it, but we suggest that the phase behaviour should not be interpreted as a running wave, rather as the result of the varying relation between the phases of the magnetic and acoustic parts of the magneto-acoustic mode in this high layer.

Much more interesting is the behaviour of the amplitudes and phases for the 4.030$\mathrm{mHz}$ harmonic, shown in Fig. 6 , for $\lambda 6145.070$. For this frequency the amplitude decreases strongly with height, which is not expected for a standing wave with a node below, as is seen for the 2.015-mHz mode in the left panels of Fig. 6 . This suggests another node near the top of the line-forming layer for the $4.030-\mathrm{mHz}$ frequency. Whether this frequency is an harmonic or an independent mode, it provides new, independent constraints on the atmospheric structure of $33 \mathrm{Lib}$.

Fig. 6 shows that the phases of the $4.030-\mathrm{mHz}$ harmonic go smoothly through at least $\pi$ radian change from the top to the bottom of the layer in which this line forms, and even through a full $2 \pi$ radians if the more uncertain phases from two cuts at bisector intensity 0.56 and 0.58 can be trusted. If the $4.030-\mathrm{mHz}$ frequency is from an independent mode, then we suggest that this phase change is a result of interaction between the acoustic and magnetic components of the pulsation mode, although there are no detailed models yet to test this suggestion in the theories of Cunha \& Gough (2000), Bigot et al. (2000) and Saio \& Gautschy (2004). If it is an harmonic, then this phase behaviour is also yet to be modelled. In either case there is unprecedented depth information about the atmospheric structure, the radial part of the mode or modes and the interaction of the pulsation with the magnetic field.

\section{References}

Baldry, I.K., Bedding, T.R. 2000, MNRAS, 318, 341

Baldry, I.K., Viskum, M., Bedding, T.R., Kjeldsen, H., Frandsen, S. 1999, MNRAS, 302, 381

Balona, L.A., Laney, C.D. 2003, MNRAS, 344, 242

Balona, L.A., Zima, W. 2002, MNRAS, 336, 873

Bigot, L., Dziembowski, W.A. 2002, A\&A, 391, 235

Bigot, L., Kurtz, D.W. 2004, A\&A, submitted

Bigot, L., Provost, J., Berthomieu, G., Dziembowski, W.A., Goode, P.R. 2000, A\&A, 356, 218

Cowley, C.R., Hubrig, S., Ryabchikova, T.A., Mathys, G., Piskunov, N., Mittermayer, P. 2001, A\&A, 367, 939

Cunha, M.S. 2002, MNRAS, 333, 47

Cunha, M.S., Gough, D.O. 2000, MNRAS, 319, 1020

Elkin, V.G., Riley, J., Cunha, M.S., Kurtz, D.W., Mathys, G. 2004, in preparation

Hubrig, S., Kharchenko, N., Mathys, G., North, P. 2000, A\&A, 355, 1031

Kochukhov, O., Bagnulo, S., Barklem, P.S. 2002, ApJ, 578, L75

Kochukhov, O., Ryabchikova, T. 2001a, A\&A, 377, L22

Kochukhov, O., Ryabchikova, T. 2001b, A\&A, 374, 615

Kochukhov, O. 2005, These Proceedings, GP20

Kurtz, D.W. 1982, MNRAS, 200, 807

Kurtz, D.W. 1991, MNRAS, 249, 468

Kurtz, D.W., Elkin, V.G., Mathys, G. 2003, MNRAS, 343, L5

Kurtz, D.W., Elkin, V.G., Mathys, G. 2004, submitted

Matthews, J.M., Kurtz, D.W., Martinez, P. 1999, ApJ, 511, 422

Mkrtichian, D.E., Hatzes, A.P., Kanaan, A. 2003, MNRAS, 345, 781

Ryabchikova, T., Piskunov, N., Kochukhov, O., Tsymbal, V., Mittermayer, P., Weiss, W.W. 2002, A\&A, 384, 545 
Sachkov, M., Ryabchikova, T., Kochukhov, O., Weiss, W.W., Reegen, P., Landstreet, J.D. 2004, ASP Conf. Ser., Vol. 310, Variable Stars in the Local Group, eds D.W. Kurtz \& K.R. Pollard, San Francisco ASP, 208

Saio, H., Gautschy, A. 2004, MNRAS, 350, 485

Shibahashi, H., Kurtz, D., Kambe, E., Gough, D. 2004, ASP Conf. Ser., Vol. 310, Variable Stars in the Local Group, eds D.W. Kurtz \& K.R. Pollard, San Francisco ASP, 287 